Published in final edited form as:

Clin Liver Dis. 2019 May ; 23(2): 363-382. doi:10.1016/j.cld.2019.01.001.

\title{
Obstructive Sleep Apnea and the Liver
}

\author{
Malav P. Parikha ${ }^{a}$ Niyati M. Gupta ${ }^{a}$, and Arthur J. McCullough ${ }^{a, b,{ }^{*}}$ \\ aDepartment of Gastroenterology and Hepatology, Digestive Disease and Surgery Institute, \\ Cleveland Clinic Foundation, Cleveland, $\mathrm{OH}$ 44114, USA; \\ ${ }^{b}$ Department of Inflammation and Immunity, Cleveland Clinic Lerner College of Medicine, Case \\ Western University, Cleveland, OH 44195, USA
}

\section{Keywords}

Nonalcoholic fatty liver disease; Hepatic steatosis; Obstructive sleep apnea; Chronic intermittent hypoxia and insulin resistance

\section{INTRODUCTION}

Nonalcoholic fatty liver disease (NAFLD) represents a spectrum of liver disorders, including the initial stage of simple fat accumulation (hepatic steatosis); followed by inflammatory changes leading to nonalcoholic steatohepatitis (NASH); and, finally, fibrosis and scaring resulting in liver cirrhosis and its consequences. ${ }^{1}$

The current prevalence of NAFLD is estimated to be $20 \%$ to $30 \%, 2,3$ and it has become the second most common indication for liver transplantation in the United States, after chronic hepatitis $\mathrm{C} .{ }^{4}$ It is projected that over the next 15 years, NASH will become the most common disease cause for liver transplantation. ${ }^{5}$ This exponential increase in the incidence and progression of NASH is attributed to the worldwide epidemic of obesity, ${ }^{6}$ which has also resulted in an increase in the prevalence of other obesity-related disorders, including metabolic syndrome, type 2 diabetes mellitus (DM), cardiovascular disease, and obstructive sleep apnea (OSA) ${ }^{7}$

OSA is caused by complete or partial obstruction of the upper airway. This results in repetitive episodes of shallow or paused breathing during sleep and causes a reduction in blood oxygen saturation. This nocturnal hypoxia, or chronic intermittent hypoxia (CIH), is the most important factor linking OSA and NAFLD. ${ }^{8-10}$ Recent studies have conclusively shown the role of OSA in the development and progression of NAFLD in terms of liver enzyme elevation and histologic alterations (steatosis, lobular inflammation, ballooning degeneration, and fibrosis). ${ }^{11-19}$ This article discusses the pathologic mechanisms associating OSA with NAFLD and the impact of OSA treatment on NAFLD outcomes.

*Corresponding author. mcculla@ccf.org.

Disclosure Statement: The authors have nothing to disclose. 


\section{OBSTRUCTIVE SLEEP APNEA}

OSA is a common clinical condition in which the throat narrows or collapses repeatedly during sleep, resulting in episodes of intermittent oxygen desaturations and nocturnal awakenings. ${ }^{20,21} \mathrm{It}$ is estimated to be present in $4 \%$ to $5 \%$ of the general population and is seen twice as commonly in men as in women. Advancing age, male gender, obesity, neck thickness, craniofacial changes, and upper airway soft tissue abnormalities are important risk factors for OSA. ${ }^{20-22}$

The direct consequences of airway collapse are snoring; increased respiratory efforts; hypercapnia; and, most importantly, CIH. This hypoxemia is sensed by carotid body receptors, leading to sympathetic activation; arousal; clearing of the airway; and, eventually, reoxygenation. The cycle of deoxygenation and reoxygenation is repeated several times every night and results in increased catecholamine release, reactive oxygen species (ROS) generation, oxidative stress, and a state of systemic inflammation. Lack of a restorative night sleep also results in excessive daytime sleepiness; morning headaches; concentration difficulties; anxiety; depression; road-traffic accidents; and, in general, a poor quality of life. $20,21,23$ OSA is also associated with hypertension, atherosclerosis, coronary artery disease, stroke, insulin resistance, and NAFLD. ${ }^{24-27}$

Polysomnography is the gold standard test for diagnosing OSA and involves recording of several physiologic parameters, including electroencephalogram, electrooculogram, and electromyogram, along with nasal and oral airflow measurements. ${ }^{28}$ An episode of apnea is defined by cessation of airflow for greater than 10 seconds despite ongoing inspiratory effort; whereas an episode of hypopnea is defined by greater than $50 \%$ reduction in airflow or moderate airflow reduction ( $<50 \%)$ along with either desaturation or electroencephalographic evidence of awakening. The severity of sleep apnea is characterized by the apnea-hypopnea index (AHI), which is simply calculated by dividing the number of events by number of hours of sleep. Accordingly, OSA can be classified as mild (AHI: 515), moderate (15-30), and severe (>30). ${ }^{29}$ Continuous positive airway pressure (CPAP) is the first-line treatment for OSA. It results in more restful sleep, reduced daytime symptoms, and improved quality of life. ${ }^{30}$ However, the effect of CPAP therapy on other chronic conditions, including metabolic syndrome and NAFLD, is less clear (see later discussion).

\section{PATHOGENESIS OF NONALCOHOLIC STEATOHEPATITIS}

\section{Two-Hit Hypothesis}

Berson and colleagues ${ }^{31}$ conducted a pivotal study, during which rat liver mitochondria and rat hepatocytes were exposed to a hepatotoxic drug $4,4^{\prime}$-diethylaminoethoxyhexestrol. This resulted in hepatic steatosis and inhibition of mitochondrial $\beta$-oxidation. Inhibition of mitochondrial respiration caused reduced adenosine triphosphate (ATP) levels and raised levels of ROS. The increased oxidative stress resulted in lipid peroxidation and subsequent cell death.

Day and James ${ }^{32}$ proposed the popular 2-hit hypothesis (Fig. 1) of steatohepatitis based on this study. ${ }^{31}$ The first hit represents hepatic steatosis, which could be related to factors such 
as excess caloric intake, obesity, or insulin resistance. A subsequent second hit, in the form of an oxidative stress, increased lipid peroxidation, and activation of inflammatory cascade, causes progression to steatohepatitis and fibrosis.

Multiple-hit hypothesis-Although the 2-hit hypothesis remains extremely popular and is often cited, emerging data show that it is too simplistic to explain the complex interplay of the multiple factors involved in the development of NASH. An alternative a multiple-hit hypothesis has been proposed that attempts to take into account several of the underlying mechanisms that may contribute to the pathogenesis of NASH. ${ }^{33,34}$

Genetic predisposition, environmental factors, and dietary habits lead to development of obesity, metabolic syndrome, and insulin resistance. Insulin resistance is a key factor in the progression of NAFLD because it not only leads to increased peripheral lipolysis with increased flux of free fatty acids (FFAs) but also to hepatic de novo lipogenesis (DNL). It also causes adipose tissue dysfunction with altered secretion of adipokines and increased levels of inflammatory cytokines, interleukin (IL)-6, and tumor necrosis factor (TNF)-a. ${ }^{35}$ Alteration of gut microbiome causes increased gut permeability, systemic levels of lipopolysaccharides, and absorption of FFAs. ${ }^{17}$

All of these factors cause an increased flux of FFAs into the liver. This results in excess triglyceride (TG) deposition in the liver (hepatic steatosis) that parallels the generation of lipotoxic metabolites of FFAs. Further, these toxic metabolites cause mitochondrial dysfunction with increased oxidative stress, generation of ROS, and endoplasmic reticulum stress, which manifests in the form of hepatocyte injury and inflammation. Hence, TG accumulation in the hepatocyte is just an innocent bystander or an epiphenomenon in NAFLD pathogenesis, with the toxic metabolites of FFAs being the major mechanism for hepatotoxicity. ${ }^{33,34}$

\section{LINK BETWEEN OBSTRUCTIVE SLEEP APNEA AND NONALCOHOLIC FATTY LIVER DISEASE}

Several studies have firmly established the relationship between OSA and NAFLD in adult and pediatric populations (Table 1). The severity of sleep apnea and, particularly, its manifestation, $\mathrm{CIH}$, is the most important trigger for increased oxidative stress, generation of ROS, and release of inflammatory cytokines, resulting systemic inflammation that drives the exacerbation of NAFLD and progression to liver fibrosis. ${ }^{10} \mathrm{CIH}$ causes reduced oxygen tension in the liver, particularly in the hepatocytes surrounding the central vein (zone 3) and results in the expression of hypoxia inducible factors (HIFs), which are the key oxygen sensors that mediate the ability of the cell to respond to a hypoxic environment. HIFs are implicated in the development of dyslipidemia, hepatic steatosis, insulin resistance, and liver fibrosis, and are a key link in the association of OSA and NAFLD. ${ }^{10,50}$

\section{Obstructive Sleep Apnea and Dyslipidemia}

Increased de novo lipogenesis-Entry of lipid substrates in the liver occurs through 1 of the following 3 mechanisms:(1) dietary intake of lipids and carbohydrates, (2) de novo 
lipogenesis, or (3) flux of FFAs from peripheral lipolysis, whereas liver disposes lipids through (1) storage as TGs, (2) oxidation of FAAs, or (3) export of TGs as very-low density lipoproteins (VLDLs) in the peripheral blood (Fig. 2) ${ }^{51}$ Studies have identified several regulators that control hepatic lipid metabolism. Sterol receptor element-binding protein (SREBP) is a transcription factor that plays a vital role in hepatic DNL. ${ }^{51,52}$ It consists of 3 isoforms, of which SREBP- $1_{c}$ is predominantly expressed in liver. It mediates the expression of lipogenic genes such as fatty acid synthase and acyl-CoA carboxylase and thereby promotes de novo FFAs and TG synthesis. ${ }^{55}$ It also increases stearoylcoenzyme-A desaturase (SCD)-1 activity that is responsible for converting polyun-saturated fatty acids into monounsaturated fatty acids (MUFAs). MUFAs are converted into cholesterol esters and TGs, which are incorporated into secreted particles..$^{53}$

$\mathrm{CIH}$, a major component of OSA, is independently associated with dyslipidemia in NAFLD. 53,54 Studies in mice have shown that exposure to CIH results in enhanced expression of previously mentioned lipogenic genes, resulting in higher TG content in the liver. ${ }^{55}$ On the contrary, interruption of SREBP-1 signaling and depletion of SCD-1 prevents hyperlipidemia during CIH. ${ }^{56,57}$ In a study by Li and colleagues, ${ }^{58}$ under hypoxic conditions, protein levels of nuclear isoforms of SREBP-1 and SCD-1 were significantly lower in mice with partial deficiency of HIF-1a compared with wild-type mice. As a result, mice with partial deficiency of HIF-1a were protected against hypertriglyceridemia and hepatic fat accumulation during CIH. HIF-1a is a master regulator of metabolic responses to hypoxia and these data confirm that $\mathrm{CIH}$ increases lipogenesis through the mediation of HIF-1a.

\section{Enhanced peripheral lipolysis and reduced lipoprotein clearance-CIH raises} sympathetic activity and induces a state of insulin resistance. This promotes lipolysis in the adipose tissue and increased flux of FFAs in the liver. Under normoxia conditions, FFAs are metabolized by oxygen-dependent mitochondrial combustion through $\beta$-oxidation. Hence hypoxia creates a condition of excess FFAs and its reduced utilization through mitochondrial $\beta$-oxidation. More FFAs become available for TG and cholesterol synthesis, which eventually results in fatty liver, liver injury through oxidative stress, and NASH. CIH has also been shown to selectively inactivate the adipose tissue lipoprotein lipase and reduce the clearance of VLDL from circulation. ${ }^{59}$ In summary, CIH can cause dyslipidemia by upregulating DNL and lipoprotein secretion, and reducing lipoprotein clearance, along with enhanced peripheral lipolysis and influx of FFAs in the liver.

\section{Obstructive Sleep Apnea and Insulin Resistance}

OSA is associated with $\mathrm{CIH}$ and sleep fragmentation, and an increasing pool of evidence now points toward an association between OSA, insulin resistance, and predisposition to type 2 DM (Fig. 3). In a study by Stamatakis and Punjabi, ${ }^{60}$ sleep was experimentally fragmented across all stages using auditory and mechanical stimuli in healthy normal volunteers. After 2 nights of sleep fragmentation, they were noted to have reduced insulin sensitivity and glucose effectiveness with an elevated morning cortisol level and increased sympathetic tone. Increase in cortisol levels ${ }^{61}$ and raised sympathetic tone ${ }^{62,63}$ are well known to promote insulin resistance by reduction of insulin secretion from the pancreas, 
inhibition of insulin-mediated glucose uptake, and increased hepatic gluconeogenesis. In another study, selective suppression of slow-wave sleep in young healthy adults showed similar results. Sensitivity to insulin was markedly reduced without adequate compensatory increase in insulin release, leading to reduced glucose tolerance and increased DM risk. ${ }^{64} \mathrm{In}$ a study by Ip and colleagues, ${ }^{65} 270$ consecutive subjects referred for suspected sleep apnea and no underlying type $2 \mathrm{DM}$ were included and tested for insulin resistance. OSA was associated with insulin resistance as measured by HOMA-IR, independent of body mass index, and progressive increase in insulin resistance was noted per each additional apnea or hypopnea per sleep hour. Mouse models of OSA have further implicated the role of ROS, ${ }^{66}$ pancreatic beta cell apoptosis, ${ }^{67}$ and inflammation ${ }^{68}$ in the development of insulin resistance and predisposition to type 2 DM. ${ }^{60}$ Hence, several human and animal studies establish a robust association between OSA and insulin resistance.

Insulin resistance is known to play a crucial role in the pathogenesis of NAFLD. Studies have shown increased adipose tissue and hepatic insulin resistance, and reduced whole-body sensitivity to insulin in NAFLD patients. These are manifested by increased peripheral lipolysis, impaired inhibition of hepatic gluconeogenesis, and reduced glucose disposal, respectively. Further, inability of the insulin to suppress peripheral lipolysis results in increased flux of FFAs to liver and contributes to hepatic DNL. Insulin resistance leads to a state of hyperinsulinemia, which stimulates lipogenic genes via SREBP-1c and further contributes to hepatic steatosis. Overall, hepatic DNL, increased flux of FFAs, and impaired mitochondrial oxidation of FFAs creates a perfect milieu for development and progression of NAFLD.

\section{Obstructive Sleep Apnea and Adipose Tissue Dysfunction}

Traditionally considered an inert tissue for pure energy storage, it now clear that adi-pose tissue is a major endocrine and a signaling organ. During obesity, hypertrophied adipocytes mediate inflammation and harbor an increased proportion of proinflammatory macrophages compared with the antiinflammatory type. Secretion of adiponectin, which mediates a protective role in NAFLD by improving insulin sensitivity and regulating fatty acid oxidation, is reduced. In contrast, the release of proinflammatory cytokines such as TNF-a and IL-6 is increased, which reduces hepatic insulin sensitivity. Peripheral lipolysis is also increased, along with the flux of FFAs to the liver, which further potentiates hepatic and muscle insulin resistance ${ }^{69-71}$ It has been conclusively proven in animal models that during obesity adipose tissue is hypoxic and the local adipose tissue hypoxia is responsible for the dysregulated production of adipokines and metabolic syndrome. ${ }^{69}$

Because inflammation and hypoxia play crucial roles in obesity-mediated adipose tissue dysfunction, $\mathrm{CIH}$, as it occurs in OSA, can be postulated to further intensify adipose tissue dysfunction. To this effect, various animal studies and models have conclusively established the role of intermittent hypoxia (IH) in inducing adipose tissue inflammation and dysfunction, even in the absence of obesity. ${ }^{70,72}$ In a recent study by Taylor and colleagues, ${ }^{73}$ human adipocytes exposed to IH showed increase in nuclear factor- $\kappa \beta$ DNA-binding activity compared with controls. There was also a significant increase in the secretion of inflammatory cytokines such as IL-8, IL-6, and TNF- $a$ with IH in adipocytes. Hence, it was 
concluded that human adipocytes are sensitive to IH, which enhances the expression of inflammatory genes and the release of inflammatory cytokines. Overall, these data provide evidence that IH can mediate adipose tissue dysfunction and the release of proinflammatory adipokines, which are known to be involved in pathogenesis of NAFLD. However, further studies are required in humans to understand the exact underlying mechanisms of IH, especially the impact of obesity.

\section{Obstructive Sleep Apnea and Mitochondrial Dysfunction}

Mitochondria are responsible for the production of 95\% the cellular energy source, ATP. Under aerobic conditions, mitochondria produce ATP via 3 main biochemical pathways: the tricarboxylic acid cycle or Krebs cycle, oxidative phosphorylation, and fatty acid $\beta$ oxidation. ${ }^{74}$ In OSA, hypoxia, along with an increased oxidative stress and flux of FAAs, overwhelm these normal mechanisms and result in structural and functional alteration of the mitochondria. Structural alteration is characterized by depletion of mitochondrial DNA ${ }^{75}$ and upregulated transcriptional and replication machinery of mitochondrial biogenesis. ${ }^{76}$ Increased levels of TNF- $\mathrm{a}$, ROS, and lipid peroxidation products alter the mitochondrial respiratory chain, block the flow of electrons in the respiratory chain, and increase the mitochondrial ROS formation. The resultant oxidative stress further activates inflammatory pathways, contributing to hepatocytes inflammation and the diverse hepatic lesions of NASH. ${ }^{77}$

\section{Obstructive Sleep Apnea and Liver Fibrosis}

Stellate cells and portal fibroblasts are important sources of fibrillar collagen and lysyl oxidase (LOX) enzymes in the normal liver and after early hepatic injury (Fig. 4) ${ }^{78}$ Hypoxia is a potent stimulator of LOX activity, which in turn plays an important role in the covalent cross-linking of collagen and elastin, increasing liver stiffness. ${ }^{79}$ This increased stiffness causes increased mechanical tension that is crucial for the differentiation of hepatic stellate cell and portal fibroblasts into myofibroblasts, which are responsible for deposition of extracellular collagen and, eventually, the development of fibrosis. ${ }^{78}$ Mesarwi and colleagues ${ }^{80}$ have recently demonstrated that serum LOX is elevated in patients with NAFLD-associated hepatic fibrosis, relative to those without fibrosis. These same investigators also proposed the potential role of serum LOX as a biomarker of liver fibrosis in patients with severe obesity and OSA. HIF-1a has also been independently implicated in the development of liver fibrosis in a mouse model of NAFLD. ${ }^{81}$ Hence, it can be concluded that hypoxia induces HIF-1a, which in turn induces the expression of LOX enzyme and the subsequent development of fibrosis.

\section{Continuous positive airway pressure treatment and impact on nonalcoholic} fatty liver disease-CPAP was originally described in 1983 by Sullivan and colleagues ${ }^{82}$ and is considered the gold standard therapy for moderate to severe OSA. CPAP therapy acts as a pneumatic support, causing the pharyngeal intraluminal pressure to exceed the surrounding pressure. It also stabilizes the upper airway by increasing the end-expiratory lung volume, thereby preventing the hypoxic events related to the upper airway collapse. ${ }^{83}$ Studies have conclusively established the benefits CPAP therapy in decreasing the hypoxic events and daytime sleepiness, lowering the risk of motor-vehicle accidents, and improving 
hypertension and a better quality of life in general. ${ }^{84}$ However, studies have yielded conflicting results about the efficacy of CPAP therapy on metabolic syndrome, including insulin resistance, lipid profile, and body fat composition. ${ }^{85,86}$

Given that CIH plays a vital role in mediation of NAFLD in OSA, treatment with CPAP would be expected to yield unequivocal benefits in NAFLD patients. However, the available studies have yielded mixed results and are listed in Table 2 and Table $3 .{ }^{80,87-102}$ In these studies, the impact of CPAP therapy on NAFLD was assessed by means of improvements in liver enzymes, hepatic adiposity, or fibrosis. Importantly, the observational studies that demonstrated the benefits of CPAP (see Table 2) were of longer duration than the randomized controlled trials that did not show CPAP to be beneficial (see Table 3). Progression of liver fibrosis from 1 stage to another takes an average of 7 years in patients with NASH and 14 years in those with NAFLD. ${ }^{103}$ Hence it is likely that studies of longer duration will be required to demonstrate the importance of OSA in the pathogenesis of NAFLD and the benefits of CPAP in its treatment. Increasing data indicate that CPAP should be considered as an integral component in the management off NAFLD patients with moderate to severe OSA and that more than 3 months of treatment with appropriate compliance is needed to notice any significant improvements in NAFLD parameters (see Table 2). As mentioned earlier, development of NAFLD requires multiple-hits and aberrations in multiple metabolic pathways. Hence, its effective management also needs a multi-modal approach with paramount emphasis on diet and lifestyle modification, and weight loss, with CPAP being an essential element in those with NAFLD and moderate to severe OSA. ${ }^{104}$

\section{SUMMARY}

$\mathrm{CIH}$ is an important risk factor in the pathogenesis of NAFLD in patients with moderate to severe OSA. Reduced oxygen tension induces HIFs, which are implicated in the development of dyslipidemia, hepatic steatosis, insulin resistance, and liver fibrosis, and are a key link in the association of OSA and NAFLD. Given that several metabolic pathways are involved in its pathogenesis, a multipronged approach to NAFLD management is required, with emphasis on weight loss and lifestyle modification. The role of CPAP in the management of NAFLD is yet to be established firmly; however, adequate duration of CPAP therapy with appropriate compliance are important to notice any significant improvements in NAFLD parameters.

\section{Acknowledgments}

Funded in part by NIH grant U01 DK061732.

\section{REFERENCES}

1. Ratziu V, Bellentani S, Cortez-Pinto H, et al. A position statement on NAFLD/NASH based on the EASL 2009 special conference. J Hepatol 2010;53:372-84. [PubMed: 20494470]

2. Angulo P Nonalcoholic fatty liver disease. N Engl J Med 2002;346:1221-31. [PubMed: 11961152]

3. Younossi ZM, Koenig AB, Abdelatif D, et al. Global epidemiology of nonalcoholic fatty liver disease-Meta-analytic assessment of prevalence, incidence, and outcomes. Hepatology 2016;64(1): 73-84. [PubMed: 26707365] 
4. Anstee QM, Targher G, Day CP. Progression of NAFLD to diabetes mellitus, cardiovascular disease or cirrhosis. Nat Rev Gastroenterol Hepatol 2013;10: 330-44. [PubMed: 23507799]

5. Parikh ND, Marrero WJ, Wang J, et al. Projected increase in obesity and non-alcoholicsteatohepatitis-related liver transplantation waitlist additions in the United States. Hepatology 2017 [Epub ahead of print].

6. Ogden CL, Carroll MD, Flegal KM. Prevalence of obesity in the United States. JAMA 2014;312(2): 189-90.

7. Kopelman PG. Obesity as a medical problem. Nature 2000;404(6778):635-43. [PubMed: 10766250]

8. Aron-Wisnewsky J, Minville C, Tordjman J, et al. Chronic intermittent hypoxia is a major trigger for non-alcoholic fatty liver disease in morbid obese. J Hepatol 2012;56(1):225-33. [PubMed: 21703181]

9. Dewan NA, Nieto FJ, Somers VK. Intermittent hypoxemia and OSA: implications for comorbidities. Chest 2015;147(1):266-74. [PubMed: 25560865]

10. Aron-Wisnewsky J, Clement K, Pépin JL. Nonalcoholic fatty liver disease and obstructive sleep apnea. Metabolism 2016;65(8):1124-35. [PubMed: 27324067]

11. Jin S, Jiang S, Hu A. Association between obstructive sleep apnea and non-alcoholic fatty liver disease: a systematic review and meta-analysis. Sleep Breath 2018;22(3):841-51. [PubMed: 29335916]

12. Sundaram SS, Halbower A, Pan Z, et al. Nocturnal hypoxia induced oxidative stress promotes progression of pediatric nonalcoholic fatty liver disease. J Hepatol 2016;65(3):560-9. [PubMed: 27501738]

13. Benotti P, Wood GC, Argyropoulos G, et al. Impact of obstructive sleep apnea on nonalcoholic fatty liver disease in patients with severe obesity. Obesity (Silver Spring) 2016;24:871-7. [PubMed: 26880657]

14. Corey KE, Misdraji J, Gelrud L, et al. Obstructive sleep apnea is associated with nonalcoholic steatohepatitis and advanced liver histology. Dig Dis Sci 2015; 60(8):2523-8. [PubMed: 25840922]

15. Daltro C, Cotrim HP, Alves E, et al. Nonalcoholic fatty liver disease associated with obstructive sleep apnea: just a coincidence? Obes Surg 2010;20(11): 1536-43. [PubMed: 20556538]

16. Nobili V, Alisi A, Cutrera R, et al. Altered gut liver axis and hepatic adiponectin expression in OSAS: novel mediators of liver injury in pediatric non-alcoholic fatty liver. Thorax $2015 ; 70(8)$ : 769-81. [PubMed: 26069285]

17. Nobili V, Cutrera R, Liccardo D, et al. Obstructive sleep apnea syndrome affects liver histology and inflammatory cell activation in pediatric nonalcoholic fatty liver disease, regardless of obesity/ insulin resistance. Am J Respir Crit Care Med 2014;189(1):66-76. [PubMed: 24256086]

18. Polotsky VY, Patil SP, Savransky V, et al. Obstructive sleep apnea, insulin resistance, and steatohepatitis in severe obesity. Am J Respir Crit Care Med 2009; 179(3):228-34. [PubMed: 18990675]

19. Trzepizur W, Boursier J, Mansour Y, et al. Association between severity of obstructive sleep apnea and blood markers of liver injury. Clin Gastroenterol Hepatol 2016;14(11):1657-61. [PubMed: 27155555]

20. Mannarino MR, Di Filippo F, Pirro M. Obstructive sleep apnea syndrome. Eur J Intern Med 2012;23(7):586-93. [PubMed: 22939801]

21. Lévy P, Kohler M, McNicholas WT, et al. Obstructive sleep apnea syndrome. Nat Rev Dis Primers 2015;1:15015. [PubMed: 27188535]

22. Strollo PJ Jr, Rogers RM. Obstructive sleep apnea. N Engl J Med 1996;334: 99-104. [PubMed: 8531966]

23. Azagra-Calero E, Espinar-Escalona E, Barrera-Mora JM, et al. Obstructive sleep apnea syndrome (OSAS). Review of the literature. Med Oral Patol Oral Cir Bucal 2012;17(6):e925-9. [PubMed: 22549673]

24. Jean-Louis G, Zizi F, Clark LT, et al. Obstructive sleep apnea and cardiovascular disease: role of the metabolic syndrome and its components. J Clin Sleep Med 2008;4(3):261-72. [PubMed: $18595441]$ 
25. Punjabi NM, Shahar E, Redline S, et al. Sleep-disordered breathing, glucose intolerance, and insulin resistance: the Sleep Heart Health Study. Am J Epidemiol 2004;160:521. [PubMed: 15353412]

26. Togeiro SM, Carneiro G, Ribeiro Filho FF, et al. Consequences of obstructive sleep apnea on metabolic profile: a Population-Based Survey. Obesity (Silver Spring) 2013;21:847. [PubMed: 23712988]

27. Türkay C, Ozol D, Kasapoğlu B, et al. Influence of obstructive sleep apnea on fatty liver disease: role of chronic intermittent hypoxia. Respir Care 2012;57:244. [PubMed: 21762556]

28. American Academy of Sleep Medicine. International classification of sleep disorders. 3rd edition Darien (IL): American Academy of Sleep Medicine; 2014.

29. The Report of an American Academy of Sleep Medicine Task Force. Sleep-related breathing disorders in adults: recommendations for syndrome definition and measurement techniques in clinical research. Sleep 1999;22:667-89. [PubMed: 10450601]

30. Giles TL, Lasserson TJ, Smith BJ, et al. Continuous positive airways pressure for obstructive sleep apnea in adults. Cochrane Database Syst Rev 2006;(1):CD001106.

31. Berson A, De Beco V, Lettéron P, et al. Steatohepatitis-inducing drugs cause mitochondrial dysfunction and lipid peroxidation in rat hepatocytes. Gastroenterology 1998;114(4):764-74. [PubMed: 9516397]

32. Day CP, James OF. Steatohepatitis: a tale of two "hits"? Gastroenterology 1998; 114(4):842-5. [PubMed: 9547102]

33. Buzzetti E, Pinzani M, Tsochatzis EA. The multiple-hit pathogenesis of non-alcoholic fatty liver disease (NAFLD). Metabolism 2016;65(8):1038-48. [PubMed: 26823198]

34. Neuschwander-Tetri BA. Hepatic lipotoxicity and the pathogenesis of nonalcoholic steatohepatitis: the central role of nontriglyceride fatty acid metabolites. Hepatology 2010;52(2):774-88. [PubMed: 20683968]

35. Guilherme A, Virbasius JV, Puri V, et al. Adipocyte dysfunctions linking obesity to insulin resistance and type 2 diabetes. Nat Rev Mol Cell Biol 2008;9:367-77. [PubMed: 18401346]

36. Tanne F, Gagnadoux F, Chazouilleres O, et al. Chronic liver injury during obstructive sleep apnea. Hepatology 2005;41:1290-6. [PubMed: 15915459]

37. Kallwitz ER, Herdegen J, Madura J, et al. Liver enzymes and histology in obese patients with obstructive sleep apnea. J Clin Gastroenterol 2007;41(10):918-21. [PubMed: 18090161]

38. Corey KE, Misdraji J, Zheng H, et al. The absence of obstructive sleep apnea may protect against non-alcoholic fatty liver in patients undergoing bariatric surgery. PLoS One 2013;8(5):e62504. [PubMed: 23658732]

39. Mir HM, Stepanova M, Afendy H, et al. Association of sleep disorders with nonalcoholic fatty liver disease (NAFLD): a population-based study. J Clin Exp Hepatol 2013;3(3):181-5. [PubMed: 25755498]

40. Minville C, Hilleret MN, Tamisier R, et al. Nonalcoholic fatty liver disease, nocturnal hypoxia, and endothelial function in patients with sleep apnea. Chest 2014;145(3):525-33. [PubMed: 24264333]

41. Sundaram SS, Sokol RJ, Capocelli KE, et al. Obstructive sleep apnea and hypoxemia are associated with advanced liver histology in pediatric nonalcoholic fatty liver disease. J Pediatr 2014;164(4):699-706.e1. [PubMed: 24321532]

42. Lin QC, Chen LD, Chen GP, et al. Association between nocturnal hypoxia and liver injury in the setting of nonalcoholic fatty liver disease. Sleep Breath 2015; 19(1):273-80. [PubMed: 24870112]

43. Agrawal S, Duseja A, Aggarwal A, et al. Obstructive sleep apnea is an important predictor of hepatic fibrosis in patients with nonalcoholic fatty liver disease in a tertiary care center. Hepatol Int 2015;9(2):283-91. [PubMed: 25788200]

44. Alkhouri N, Kheirandish-Gozal L, Matloob A, et al. Evaluation of circulating markers of hepatic apoptosis and inflammation in obese children with and without obstructive sleep apnea. Sleep Med 2015;16(9):1031-5. [PubMed: 26298775]

45. Petta S, Marrone O, Torres D, et al. Obstructive sleep apnea is associated with liver damage and atherosclerosis in patients with non-alcoholic fatty liver disease. PLoS One 2015;10(12):e0142210. [PubMed: 26672595] 
46. Cakmak E, Duksal F, Altinkaya E, et al. Association between the severity of nocturnal hypoxia in obstructive sleep apnea and non-alcoholic fatty liver damage. Hepat Mon 2015;15(11):e32655. [PubMed: 26834793]

47. Qi JC, Huang JC, Lin QC, et al. Relationship between obstructive sleep apnea and nonalcoholic fatty liver disease in nonobese adults. Sleep Breath 2016; 20(2):529-35. [PubMed: 26174847]

48. Asfari MM, Niyazi F, Lopez R, et al. The association of nonalcoholic steatohepatitis and obstructive sleep apnea. Eur J Gastroenterol Hepatol 2017;29(12): 1380-4. [PubMed: 28914694]

49. Ding H, Huang JF, Xie HS, et al. The association between glycometabolism and nonalcoholic fatty liver disease in patients with obstructive sleep apnea. Sleep Breath 2018 [Epub ahead of print].

50. Shin MK, Drager LF, Yao Q, et al. Metabolic consequences of high-fat diet are attenuated by suppression of HIF-1a. PLoS One 2012;7(10):e46562. [PubMed: 23049707]

51. Suzuki T, Shinjo S, Arai T, et al. Hypoxia and fatty liver. World J Gastroenterol 2014;20(41): 15087-97. [PubMed: 25386057]

52. Raghow R, Yellaturu C, Deng X, et al. SREBPs: the crossroads of physiological and pathological lipid homeostasis. Trends Endocrinol Metab 2008;19(2):65-73. [PubMed: 18291668]

53. Drager LF, Jun JC, Polotsky VY. Metabolic consequences of intermittent hypoxia: relevance to obstructive sleep apnea. Best Pract Res Clin Endocrinol Metab 2010;24(5):843-51. [PubMed: 21112030]

54. Adedayo AM, Olafiranye O, Smith D, et al. Obstructive sleep apnea and dyslipidemia: evidence and underlying mechanism. Sleep Breath 2012;18(1):13-8. [PubMed: 22903801]

55. Li J, Thorne LN, Punjabi NM, et al. Intermittent hypoxia induces hyperlipidemia in lean mice. Circ Res 2005;97(7):698-706. [PubMed: 16123334]

56. Li J, Nanayakkara A, Jun J, et al. Effect of deficiency in SREBP cleavage activating protein on lipid metabolism during intermittent hypoxia. Physiol Genomics 2007;31(2):273-80. [PubMed: 17666524]

57. Savransky V, Jun J, Li J, et al. Dyslipidemia and atherosclerosis induced by chronic intermittent hypoxia are attenuated by deficiency of stearoyl coenzyme A desaturase. Circ Res 2008;103(10): 1173-80. [PubMed: 18832746]

58. Li J, Bosch-Marce M, Nanayakkara A, et al. Altered metabolic responses to intermittent hypoxia in mice with partial deficiency of hypoxia inducible factor-1alpha. Physiol Genomics 2006;25(3): 450-7. [PubMed: 16507783]

59. Drager LF, Li J, Shin M-K, et al. Intermittent hypoxia inhibits clearance of triglyceride-rich lipoproteins and inactivates adipose lipoprotein lipase in a mouse model of sleep apnea. Eur Heart J 2012;33(6):783-90. [PubMed: 21478490]

60. Stamatakis KA, Punjabi NM. Effects of sleep fragmentation on glucose metabolism in normal subjects. Chest 2010;137(1):95-101. [PubMed: 19542260]

61. Andrews RC, Walker BR. Glucocorticoids and insulin resistance: old hormones, new targets. Clin Sci (Lond) 1999;96(5):513-23. [PubMed: 10209084]

62. Deibert DC, DeFronzo RA. Epinephrine-induced insulin resistance in man. J Clin Invest 1980;65(3):717-21. [PubMed: 6243677]

63. Avogaro A, Toffolo G, Valerio A, et al. Epinephrine exerts opposite effects on peripheral glucose disposal and glucose-stimulated insulin secretion. A stable label intravenous glucose tolerance test minimal model study. Diabetes 1996; 45(10):1373-8. [PubMed: 8826974]

64. Tasali E, Leproult R, Ehrmann DA, et al. Slow-wave sleep and the risk of type 2 diabetes in humans. Proc Natl Acad Sci U S A 2008;105(3):1044-9. [PubMed: 18172212]

65. Ip MS, Lam B, Ng MM, et al. Obstructive sleep apnea is independently associated with insulin resistance. Am J Respir Crit Care Med 2002;165(5):670-6. [PubMed: 11874812]

66. Peng YJ, Yuan G, Ramakrishnan D, et al. Heterozygous HIF-1alpha deficiency impairs carotid body-mediated systemic responses and reactive oxygen species generation in mice exposed to intermittent hypoxia. J Physiol 2006;577(Pt2):705-16. [PubMed: 16973705]

67. Xu J, Long YS, Gozal D, et al. Beta-cell death and proliferation after intermittent hypoxia: role of oxidative stress. Free Radic Biol Med 2009;46(6):783-90. [PubMed: 19133326] 
68. Ryan S, Taylor CT, McNicholas WT. Selective activation of inflammatory pathways by intermittent hypoxia in obstructive sleep apnea syndrome. Circulation 2005;112(17):2660-7. [PubMed: 16246965]

69. Hosogai N, Fukuhara A, Oshima K, et al. Adipose tissue hypoxia in obesity and its impact on adipocytokine dysregulation. Diabetes 2007;56(4):901-11. [PubMed: 17395738]

70. Ryan S Adipose tissue inflammation by intermittent hypoxia: mechanistic link between obstructive sleep apnea and metabolic dysfunction. J Physiol 2017; 595(8):2423-30. [PubMed: 27901270]

71. Trayhurn P, Wang B, Wood IS. Hypoxia and the endocrine and signaling role of white adipose tissue. Arch Physiol Biochem 2008;114(4):267-76. [PubMed: 18946787]

72. Poulain L, Thomas A, Rieusset J, et al. Visceral white fat remodeling contributes to intermittent hypoxia-induced atherogenesis. Eur Respir J 2014;43(2):513-22. [PubMed: 24072212]

73. Taylor CT, Kent BD, Crinion SJ, et al. Human adipocytes are highly sensitive to intermittent hypoxia induced NF-kappaB activity and subsequent inflammatory gene expression. Biochem Biophys Res Commun 2014;447(4):660-5. [PubMed: 24755071]

74. Sharpe AJ, McKenzie M. Mitochondrial fatty acid oxidation disorders associated with short-chain Enoyl-CoA Hydratase (ECHS1) deficiency. Cells 2018;7(6):46.

75. Kim YS, Kwak JW, Lee KE, et al. Can mitochondrial dysfunction be a predictive factor for oxidative stress in patients with obstructive sleep apnea? Antioxid Redox Signal 2014;21(9):12858. [PubMed: 24926527]

76. Lacedonia D, Carpagnano GE, Crisetti E, et al. Mitochondrial DNA alteration in obstructive sleep apnea. Respir Res 2015;16(1):47. [PubMed: 25890226]

77. Pessayre D, Fromenty B. NASH: a mitochondrial disease. J Hepatol 2005;42: 928-40. [PubMed: 15885365]

78. Kagan HM. Lysyl oxidase: mechanism, regulation and relationship to liver fibrosis. Pathol Res Pract 1994;190(9-10):910-9. [PubMed: 7899140]

79. Liu SB, Ikenaga N, Peng ZW, et al. Lysyl oxidase activity contributes to collagen stabilization during liver fibrosis progression and limits spontaneous fibrosis reversal in mice. FASEB J 2016;30(4):1599-609. [PubMed: 26700732]

80. Mesarwi OA, Shin MK, Drager LF, et al. Lysyl oxidase as a serum biomarker of liver fibrosis in patients with severe obesity and obstructive sleep apnea. Sleep 2015;38(10):1583-91. [PubMed: 26085300]

81. Mesarwi OA, Shin MK, Bevans-Fonti S, et al. Hepatocyte hypoxia inducible factor-1 mediates the development of liver fibrosis in a mouse model of nonalcoholic fatty liver disease. PLoS One 2016;11(12):e0168572. [PubMed: 28030556]

82. Sullivan C, Berthon-Jones M, Issa F. Nocturnal nasal-airway pressure for sleep apnea. N Engl J Med 1983;309:112.

83. Jordan AS, McSharry DG, Malhotra A. Adult obstructive sleep apnea. Lancet 2014;383:736. [PubMed: 23910433]

84. Kryger MH, Malhotra A. Management of obstructive sleep apnea in adults In: Collop N, editor. UpToDate, Available at: https://www.uptodate.com/contents/management-of-obstructive-sleepapnea-in-adults. Accessed November 24, 2018

85. Spicuzza L, Caruso D, Di Maria G. Obstructive sleep apnea syndrome and its management. Ther Adv Chronic Dis 2015;6(5):273-85. [PubMed: 26336596]

86. Liu X, Miao Y, Wu F, et al. Effect of CPAP therapy on liver disease in patients with OSA: a review. Sleep Breath 2018;22(4):963-72. [PubMed: 29327118]

87. Chin K, Nakamura T, Takahashi K, et al. Effects of obstructive sleep apnea syndrome on serum aminotransferase levels in obese patients. Am J Med 2003; 114(5):370-6. [PubMed: 12714126]

88. Shpirer I, Copel L, Broide E, et al. Continuous positive airway pressure improves sleep apnea associated fatty liver. Lung 2010;188(4):301-7. [PubMed: 20066542]

89. Hobzova M, Ludka O, Stepanova R, et al. Continuous positive airway pressure treatment and liver enzymes in sleep apnea patients. Sleep Med 2015;16:215-6. 
90. Kim D, Ahmed A, Kushida C. Continuous positive airway pressure therapy on nonalcoholic fatty liver disease in patients with obstructive sleep apnea. J Clin Sleep Med 2018;14(8):1315-22. [PubMed: 30092894]

91. Kohler M, Pepperell JC, Davies RJ, et al. Continuous positive airway pressure and liver enzymes in obstructive sleep apnea: data from a randomized controlled trial. Respiration 2009;78(2):141-6. [PubMed: 18984944]

92. Sivam S, Phillips CL, Trenell MI, et al. Effects of 8 weeks of continuous positive airway pressure on abdominal adiposity in obstructive sleep apnea. Eur Respir J 2012;40(4):913-8. [PubMed: 22267762]

93. Chen LD, Lin L, Zhang LJ, et al. Effect of continuous positive airway pressure on liver enzymes in obstructive sleep apnea: a meta-analysis. Clin Respir J 2018; 12(2):373-81. [PubMed: 27614004]

94. Yoshiro T, Kimihiko M, Masanori A, et al. Impacts of long-term CPAP therapy on fatty liver in male OSA patients with abdominal obesity. Eur Respir J 2014;44: S4661.

95. Buttacavoli M, Gruttad'Auria CI, Olivo M, et al. Liver steatosis and fibrosis in OSA patients after long-term CPAP treatment: a preliminary ultrasound study. Ultrasound Med Biol 2016;42(1):1049. [PubMed: 26385053]

96. Hoyos CM, Killick R, Yee BJ, et al. Cardiometabolic changes after continuous positive airway pressure for obstructive sleep apnea: a randomized sham-controlled study. Thorax 2012;67(12): 1081-9. [PubMed: 22561530]

97. Kritikou I, Basta M, Tappouni R, et al. Sleep apnea and visceral adiposity in middle-aged male and female subjects. Eur Respir J 2013;41(3):601-9. [PubMed: 22743670]

98. Jullian-Desayes I, Tamisier R, Zarski JP, et al. Impact of effective versus sham continuous positive airway pressure on liver injury in obstructive sleep apnea: data from randomized trials. Respirology 2016;21(2):378-85. [PubMed: 26567858]

99. Hang LW, Chen CF, Wang CB, et al. The association between continuous positive airway pressure therapy and liver disease development in obstructive sleep apnea/hypopnea syndrome patients: a nationwide population-based cohort study in Taiwan. Sleep Breath 2017;21(2):461-7. [PubMed: 27957696]

100. Sundaram SS, Halbower AC, Klawitter J, et al. Treating obstructive sleep apnea and chronic intermittent hypoxia improves the severity of nonalcoholic fatty liver disease in children. $\mathrm{J}$ Pediatr 2018;198:67-75.e1. [PubMed: 29752170]

101. Chen LD, Zhang LJ, Lin XJ, et al. Association between continuous positive airway pressure and serum aminotransferases in patients with obstructive sleep apnea. Eur Arch Otorhinolaryngol 2018;275(2):587-94. [PubMed: 29224042]

102. Labarca G, Cruz R, Jorquera J. Continuous positive airway pressure in patients with obstructive sleep apnea and non-alcoholic steatohepatitis: a systematic review and meta-analysis. J Clin Sleep Med 2018;14(1):133-9. [PubMed: 29151428]

103. Singh S, Allen AM, Wang Z, et al. Fibrosis progression in nonalcoholic fatty liver vs nonalcoholic steatohepatitis: a systematic review and meta-analysis of paired-biopsy studies. Clin Gastroenterol Hepatol 2014;13(4):643-54.e1-9 [quiz: e39-40]. [PubMed: 24768810]

104. Chirinos JA, Gurubhagavatula I, Teff K, et al. CPAP, weight loss, or both for obstructive sleep apnea. N Engl J Med 2014;370(24):2265-75. [PubMed: 24918371] 


\section{KEY POINTS}

- Chronic intermittent hypoxia (CIH) is the most important factor linking obstructive sleep apnea (OSA) and nonalcoholic fatty liver disease (NAFLD).

- $\quad \mathrm{CIH}$ results in a state of systemic inflammation, increased oxidative stress, insulin resistance, and dyslipidemia, predisposing to various manifestations on NAFLD.

- Even though the 2-hit theory has been a popular hypothesis to explain the pathogenesis of NAFLD, current evidence points toward a multiple-hit hypothesis involving complex interplay of environmental and dietary factors, role of insulin resistance, adipose tissue dysfunction, and altered gut microbiota in genetically predisposed subjects.

- The role of continuous positive airway pressure (CPAP) in the management of NAFLD is yet to be established firmly.

- In general, a multifaceted approach to NAFLD with emphasis on diet, life style modification, and weight loss is required, along with sufficiently longer duration of CPAP therapy and appropriate compliance in those with NAFLD and moderate to severe OSA. 


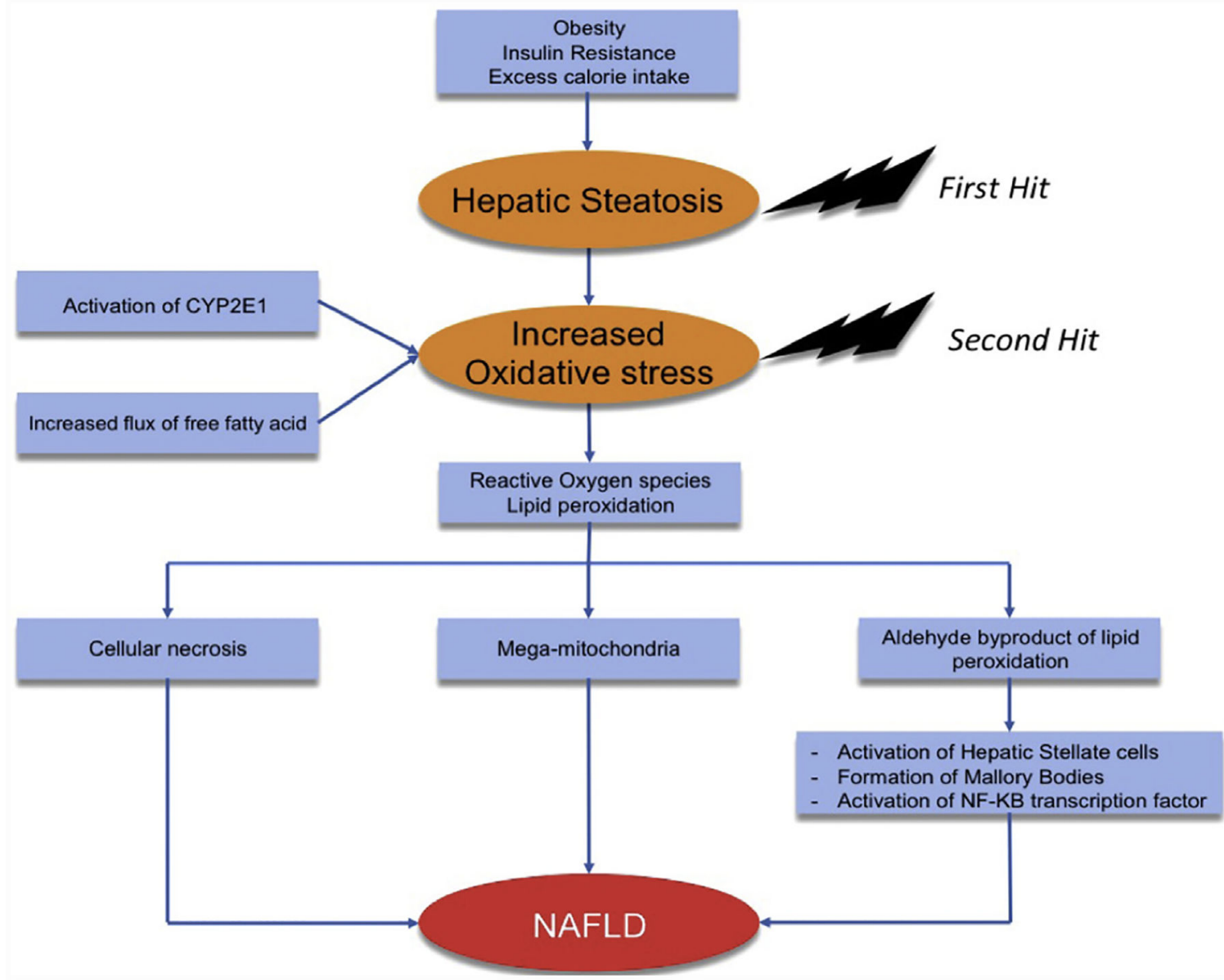

Fig. 1.

Two-hit hypothesis. CYP2E1, cytochrome P450 2E1; NF- $\kappa B$, in nuclear factor- $\kappa \beta$. 


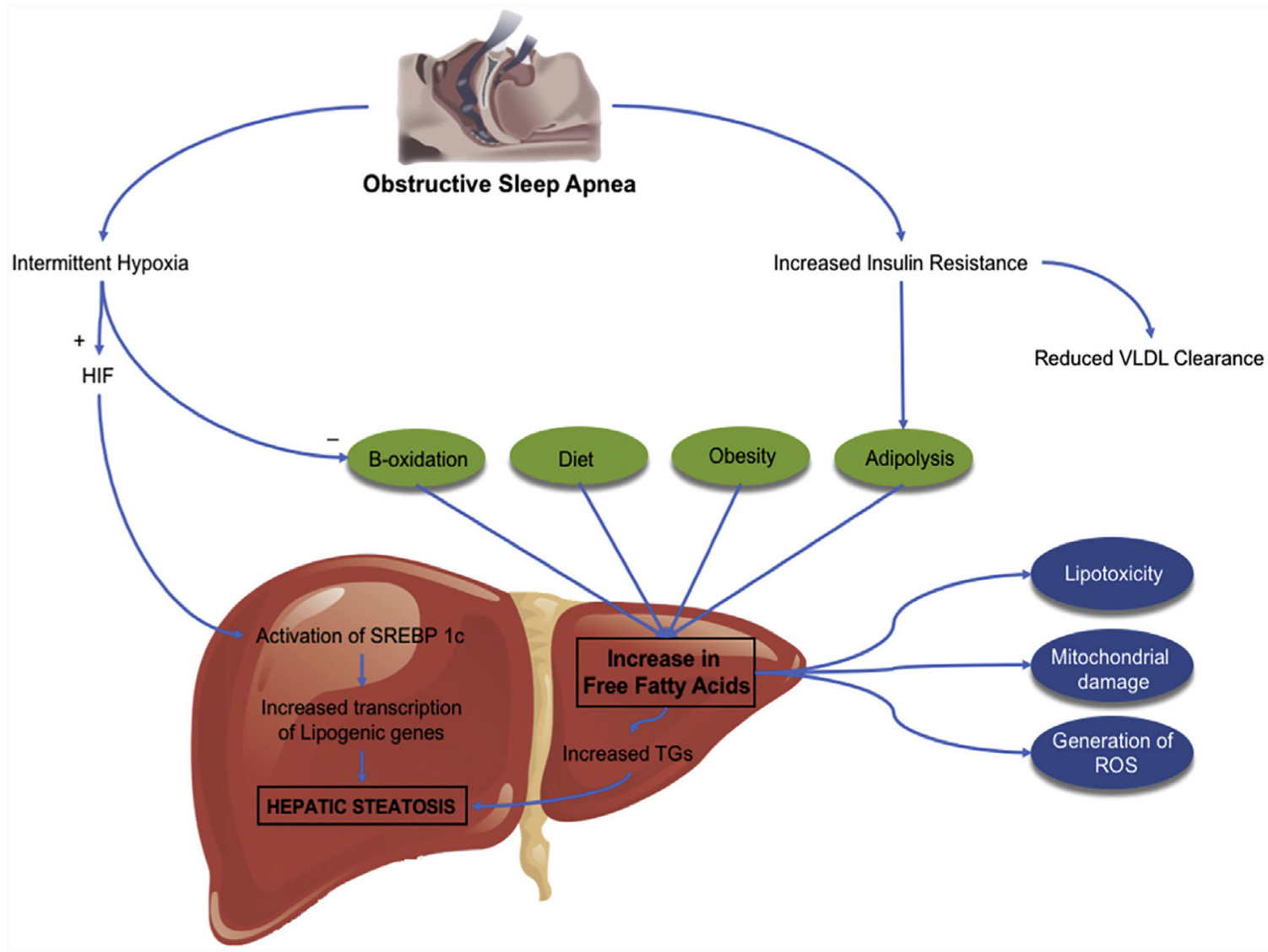

Fig. 2.

Increased de novo lipogenesis. SREBP, sterol receptor element-binding protein. 


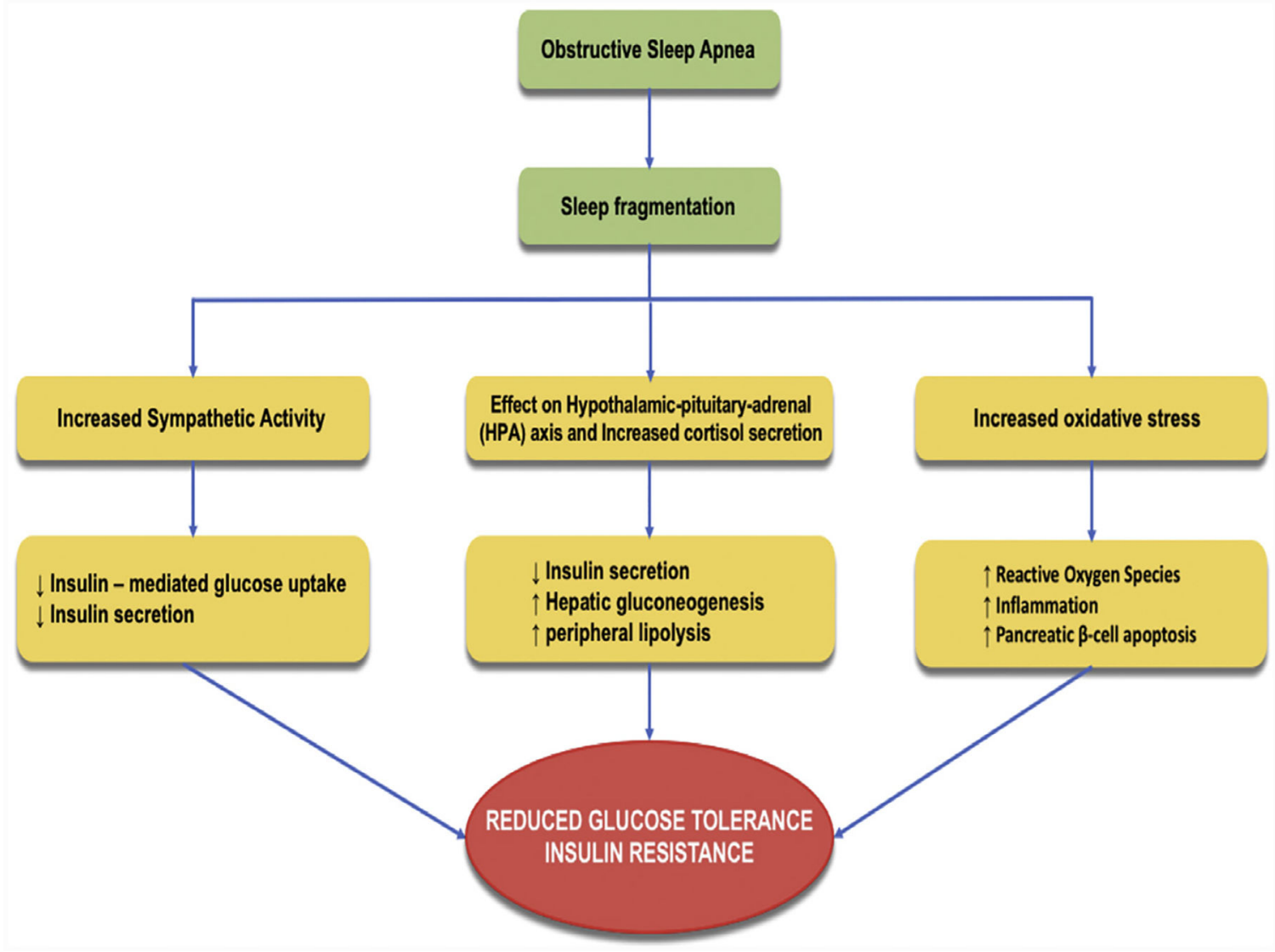

Fig. 3.

OSA and insulin resistance. 


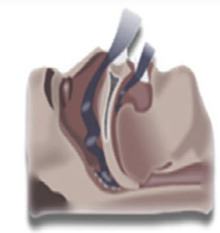

Obstructive Sleep Apnea
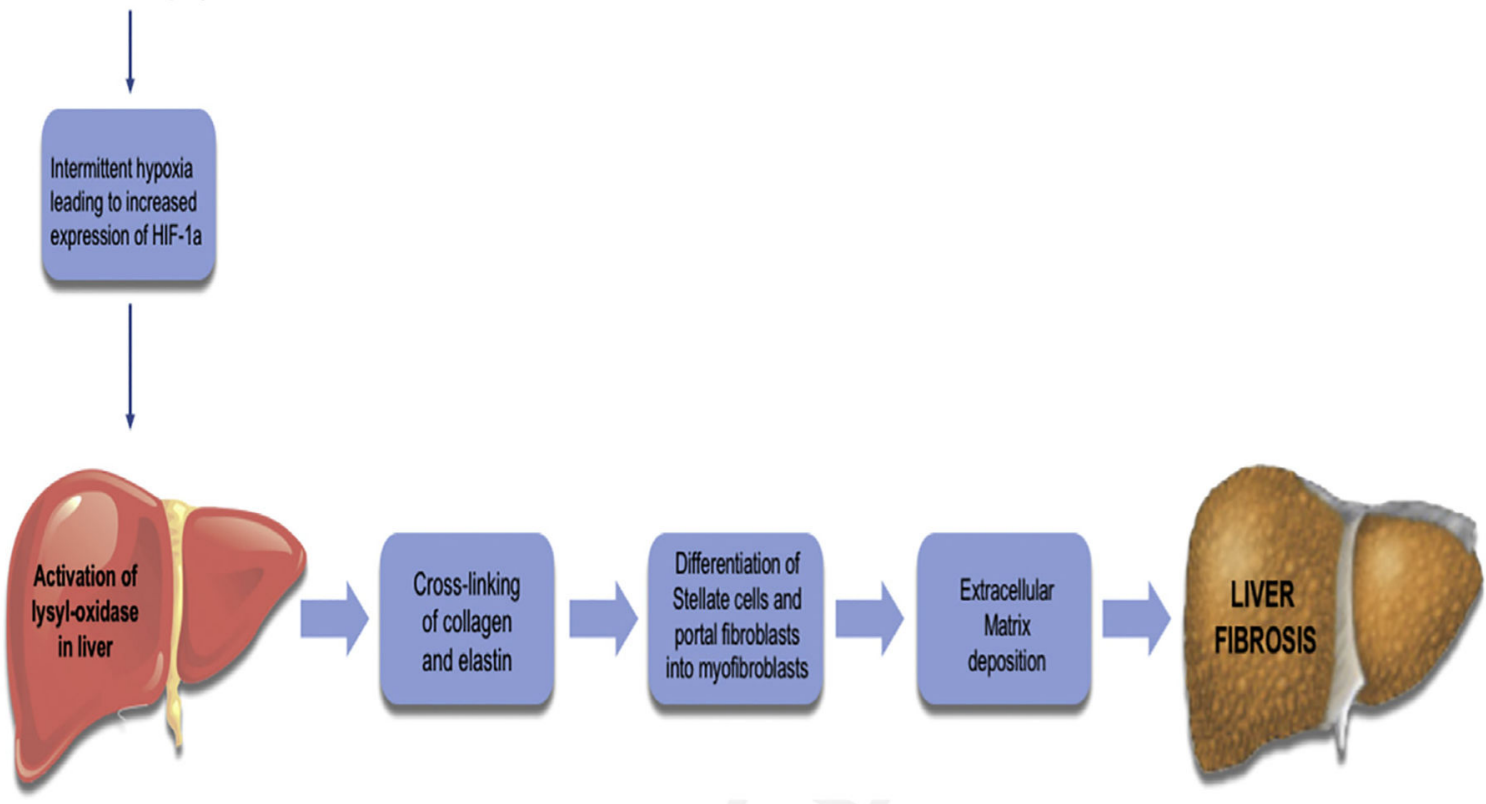

Fig. 4.

OSA and liver fibrosis. 


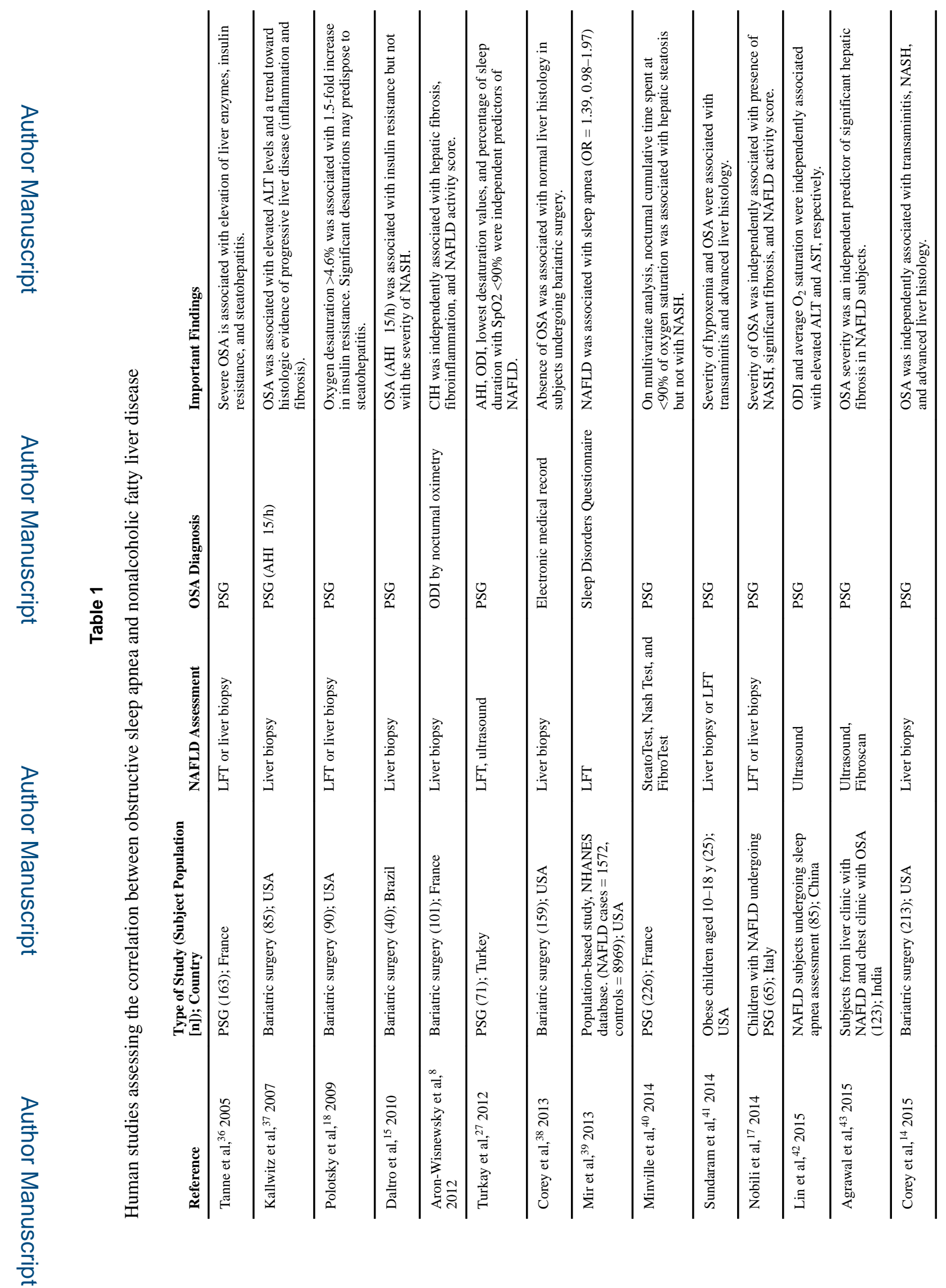

Clin Liver Dis. Author manuscript; available in PMC 2020 May 01. 
Parikh et al.

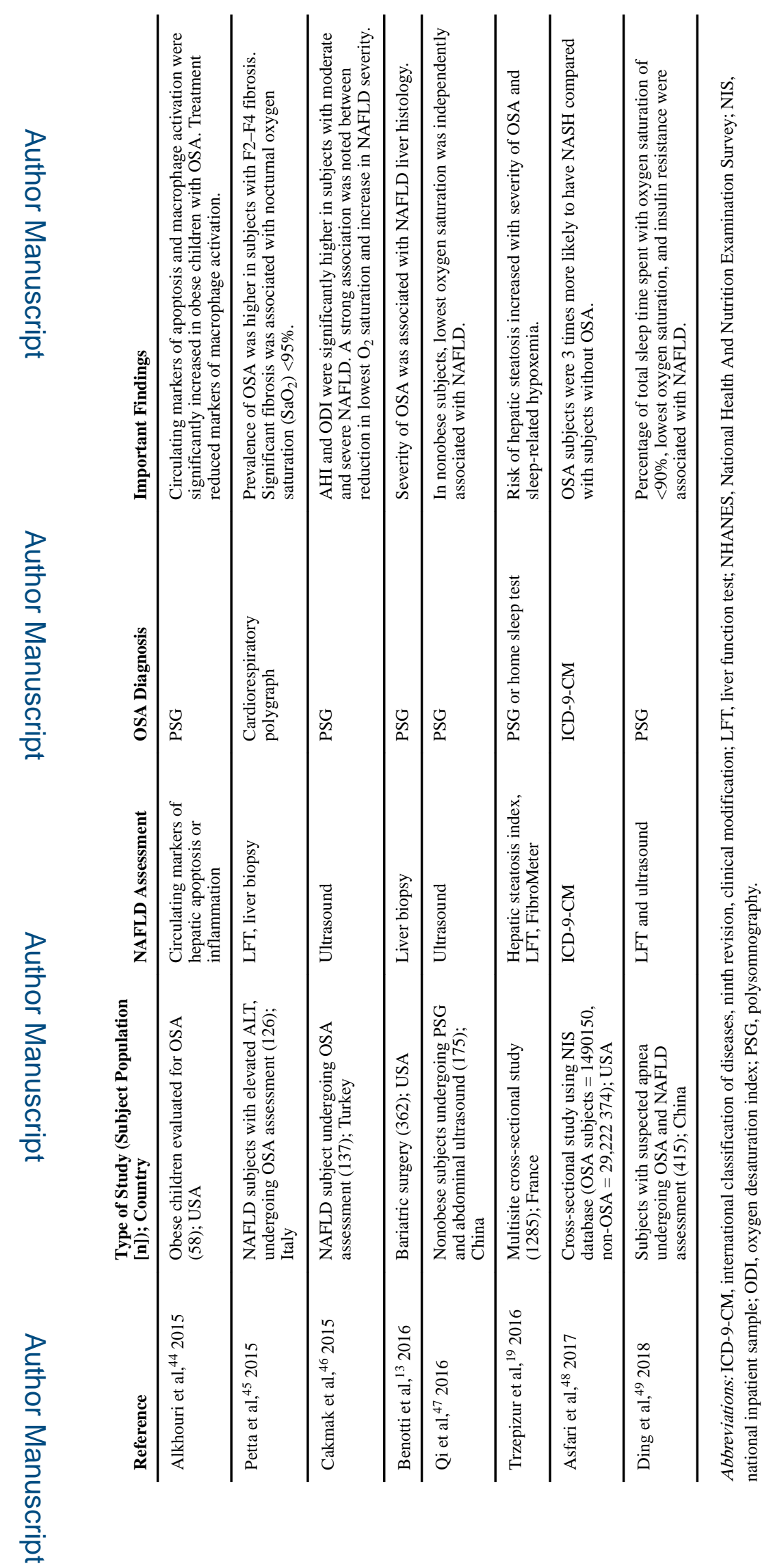

Clin Liver Dis. Author manuscript; available in PMC 2020 May 01. 
Parikh et al.

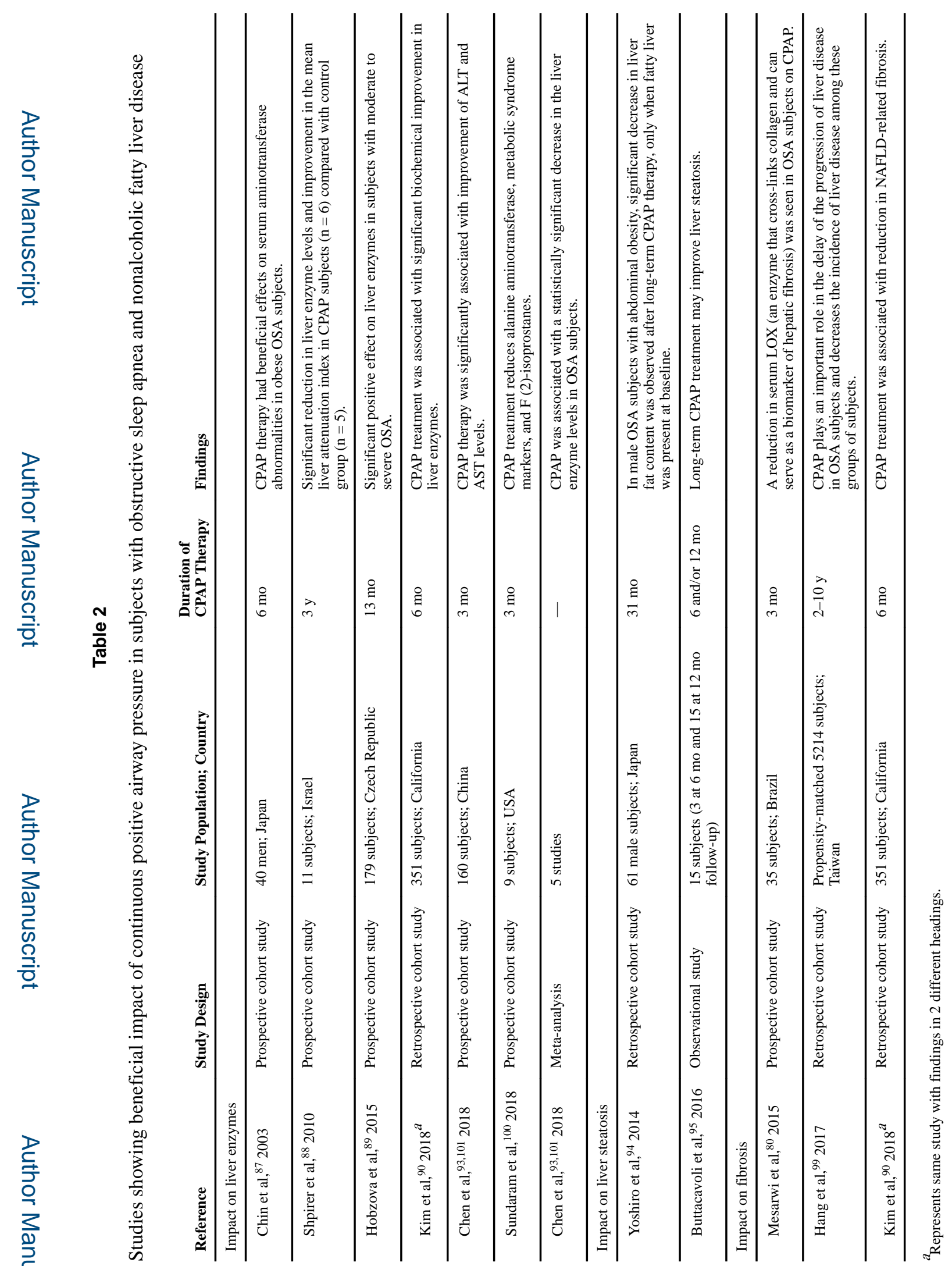

Clin Liver Dis. Author manuscript; available in PMC 2020 May 01. 
Parikh et al.

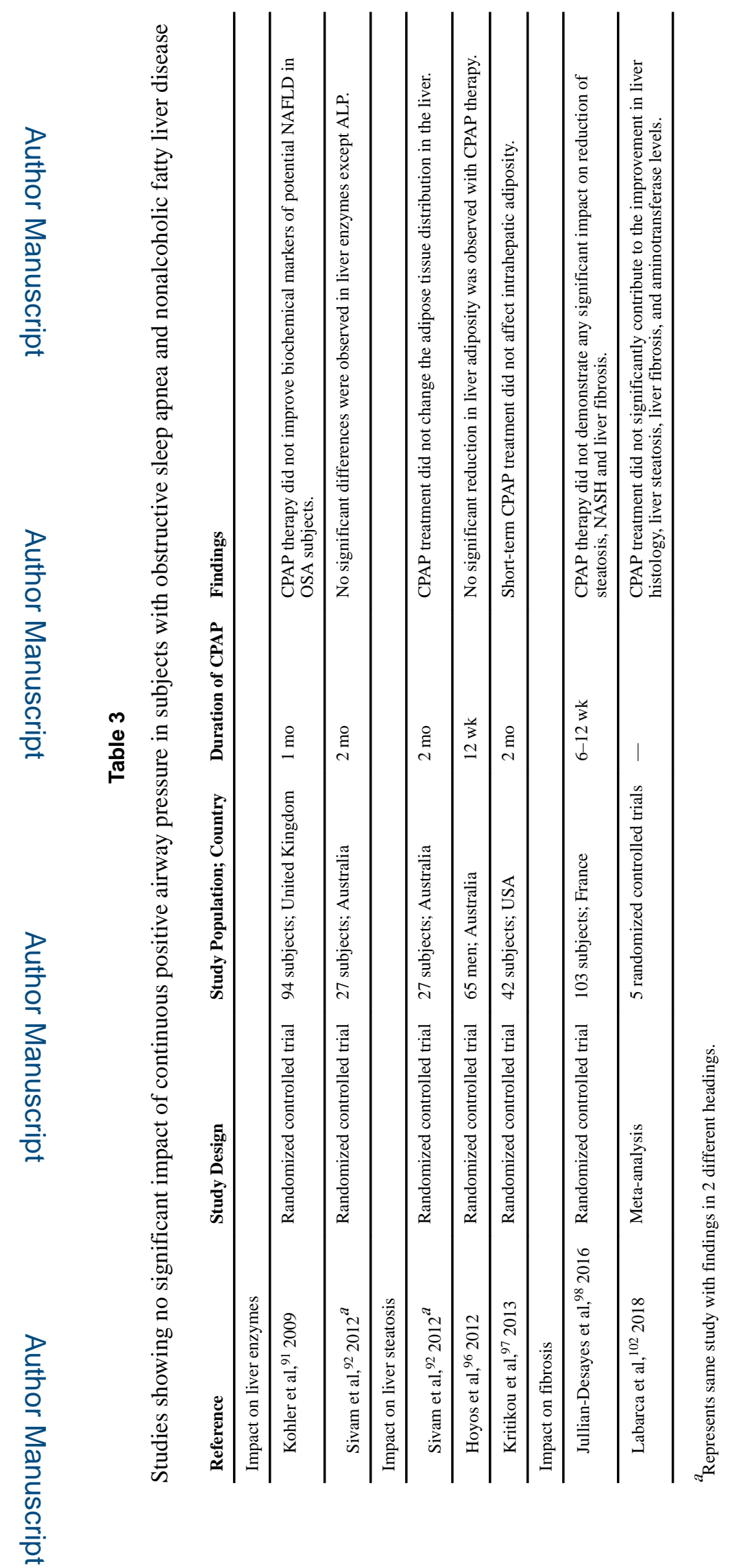

Clin Liver Dis. Author manuscript; available in PMC 2020 May 01. 